Research paper

\title{
Failure of ivermectin efficacy against Psoroptes ovis infestation in cattle: Integrated pharmacokinetic-pharmacodynamic evaluation of two commercial formulations
}

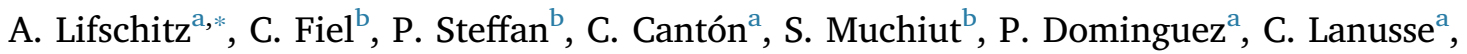 \\ L. Alvarez ${ }^{\mathrm{a}}$ \\ ${ }^{\text {a }}$ Laboratorio de Farmacología, Centro de Investigación Veterinaria de Tandil (CIVETAN) (UNCPBA-CICPBA-CONICET), Facultad de Ciencias Veterinarias, UNCPBA, \\ Tandil, Argentina \\ ${ }^{\mathbf{b}}$ Laboratorio de Parasitología y Enfermedades Parasitarias, Centro de Investigación Veterinaria de Tandil (CIVETAN) (UNCPBA-CICPBA-CONICET), Facultad de \\ Ciencias Veterinarias, UNCPBA, Tandil, Argentina
}

\section{A R T I C L E I N F O}

\section{Keywords:}

Ivermectin

Psoroptes ovis

Cattle

Pharmacokinetic-pharmacodynamic

relationship

Resistance

\begin{abstract}
A B S T R A C T
Psoroptic mange is an important parasitic disease that mainly affects beef cattle producing marked economic losses. Ivermectin (IVM) is considered one of the most effective treatments against psoroptic mange and is used worldwide to control both endo and ectoparasites in different species. The current work assessed the relationship between pharmacokinetic behavior of IVM and its efficacy against Psoroptes ovis after the subcutaneous administration of two commercial formulations in a cattle feedlot. Aberdeen Angus and Hereford steers were selected based on the presence of active mite infestations. Animals were allocated into 4 experimental groups and treated with a single (day 0) or repeated subcutaneous injection (days 0 and 7) of one of two commercial formulations of IVM (1\%) at $0.2 \mathrm{mg} / \mathrm{kg}$. Blood and skin samples were taken from 8 randomly selected animals of each experimental group to measure IVM concentrations by HPLC. Skin scrapings were also collected from six different sites in each animal, mites were counted and ranked based on a density score. Equivalent plasma concentrations of IVM were measured after the administration of IVM formulations under study. The repeated administration of both IVM formulations at day 0 and 7 accounted for a greater plasma drug availability compared with the single administration $(\mathrm{P}<0.05$ ). IVM was well distributed from the plasma to the skin without significant differences between both IVM formulations. There was a positive correlation between IVM concentrations in skin and plasma ( $\mathrm{r}: 0.73 \mathrm{P}<0.0001$ ). The mean ratios between IVM availabililty (measured as AUC) in the skin and in plasma were between 1.2 and 2.1. The repeated administration of IVM increased significantly the IVM concentrations in the skin of areas affected by mange. IVM failed to obtain a parasitological cure in the different groups affected by mange. The failure was observed with both formulations administeredat single or repeated doses. Based on the number of animals cured, the range of efficacy was between $0 \%$ on day 7 and $60 \%$ on day 28 post-treatment. No significant differences in the $P$. ovis density scores were observed after the IVM treatment at single or repeated doses. Additional studies are needed to confirm the presence of resistant strains of P.ovis and to establish the appropriate measures to control these parasitic infestations in feedlot cattle.
\end{abstract}

\section{Introduction}

Psoroptic mange caused by Psoroptes ovis is the most common type of mange in cattle and sheep in many temperate regions of the world. The disease frequently follows a severe clinical course and may lead to important losses from mortality and impaired productivity (Eddi et al., 2002). Acaricidal dips and injectable macrocyclic lactones are the main pharmacological tools recommended for controlling psoroptic scabies in cattle (Wright, 1990). Since 1980, ivermectin (IVM) has been considered one of the most effective treatments against psoroptic mange and is used worldwide to control endo and ectoparasites in different species (Campbell, 2012). The high lipophilicity of IVM and its pharmacokinetic profile support its activity against mites. After the subcutaneous treatment of cattle, IVM is extensively distributed from the

\footnotetext{
* Corresponding author at: Laboratorio de Farmacología, Centro de Investigación Veterinaria de Tandil (CIVETAN) (UNCPBA-CICPBA-CONICET), Facultad de Ciencias Veterinarias, UNCPBA, Campus Universitario, 7000 Tandil, Argentina.

E-mail address: adrianl@vet.unicen.edu.ar (A. Lifschitz).
} 
bloodstream to different target tissues including the different layers of the skin (Lifschitz et al., 2000).

Different factors may increase the risk of development of psoroptic mange: high cattle concentration, short sunlight period, cold and humid climate, low food availability and the presence of internal parasites (Eddi et al., 2002). However, in endemic areas of South America, the frequent use of IVM has reduced the number of mange outbreaks. Although mite eggs are not susceptible to the different antiparasitic drugs, the persistence of IVM in the animals leads to the death of newly born larvae and the elimination of the $P$. ovis infestation following a single injection of $0.2 \mathrm{mg} / \mathrm{kg}$ (Guillot and Meleney, 1982; Lonneux et al., 1997).

After several years of intensive use, resistance to IVM is widespread in nematodes from small ruminants and cattle (Kaplan and Vidyashankar, 2012; Cristel et al., 2017). However, information on the resistance of $P$. ovis to IVM is scarce. A differential efficacy of two formulations of IVM has been reported in a cattle feedlot (Genchi et al., 2008). Additionally, two treatments with a generic IVM preparation given at a 14-day interval failed to cure Belgian blue cattle infected with $P$. ovis (Lekimme et al., 2010), although the presence of resistant mites could not be established. The current work assessed the relationship between the IVM pharmacokinetic behavior and its efficacy against $P$. ovis infestations following the subcutaneous administration of two commercial formulations in a cattle feedlot.

\section{Materials and methods}

\subsection{Field trial and animals}

The current study was carried out in a commercial cattle feedlot farm in which 10,000 animals are usually fattened. This feedlot is located in Tandil, Buenos Aires Province, Argentina, and had a history of previous failure of IVM treatment against mange infestations, based on empirical observations. From a herd of 150 Aberdeen Angus and Hereford steers, 40 animals $(388 \pm 53 \mathrm{~kg}$ ) were selected based on the presence of active mite infestations. All steers with mange included in the study were ear tagged. Animal procedures were performed according to the Animal Welfare Policy (act 12/2013) of the Faculty of Veterinary Medicine, Universidad Nacional del Centro de la Provincia de Buenos Aires (UNCPBA), Tandil, Argentina (http://www.vet.unicen. edu.ar).

\subsection{Experimental design, treatments and sampling}

Experimental steers were assigned to one of four experimental groups using a table of random numbers (10 animals in each group). The day of allocation was designated as Day 0. Animals were individually weighed and subcutaneously treated with IVM as follows:

GROUP 1: Animals received IVM (IVOMEC ${ }^{\circ}$, Boehringer-Ingelheim, Formulation A) at a dose rate of $0.2 \mathrm{mg} / \mathrm{kg}$ on Day 0 .

GROUP 2: Animals received two injections of IVM (IVOMEC ${ }^{\circ}$, Boehringer-Ingelheim, Formulation A) at a dose rate of $0.2 \mathrm{mg} / \mathrm{kg}$ on Day 0 and Day 7, respectively.

GROUP 3: Animals received IVM (Bagomectina, Biogenesis-Bago ${ }^{\circ}$, Formulation B) at a dose rate of $0.2 \mathrm{mg} / \mathrm{kg}$ on Day 0 .

GROUP 4: Animals received two injections of IVM (BiogenesisBago , Formulation B) at a dose rate of $0.2 \mathrm{mg} / \mathrm{kg}$ on Day 0 and Day 7 , respectively.

Following treatments, the experimental groups were separated outdoors in different pens in order to avoid contact among them and with other animals in the feedlot. Water was supplied ad libitum and animals were offered a mixed ration given twice a day. The health of the animals was assessed at regular intervals throughout the study. An untreated control group was not included in the study to avoid potential and serious animal welfare issues.

\subsubsection{Pharmacological assay}

Blood and skin samples were taken from 8 randomly selected animals of each experimental group. Blood samples $(7 \mathrm{~mL})$ from the jugular vein were collected from each animal by means of heparinised Vacutainer tubes (Becton Dickinson, NJ, USA) prior to treatment and at 7, 14, 21 and 28 days post-treatment. Skin samples were also collected from the areas affected by mange prior to treatment and at 7, 14, 21 and 28 days post-treatment and kept in labeled vials. Blood samples were centrifuged at $2000 \times g$ for $20 \mathrm{~min}$ and the recovered plasma was kept in labeled vials. Plasma and skin samples were stored at $-20{ }^{\circ} \mathrm{C}$ until analysis by high-performance liquid chromatography (HPLC) to determine IVM concentrations.

\subsubsection{Parasitological observations}

On days $0,7,14,21$ and 28 post-treatment, skin scrapings were collected from the periphery of active lesions of individual animals. At each sampling time, six different sites measuring approximately $6 \mathrm{~cm}^{2}$ were scraped on each animal using a sharp spoon. Mites found on each animal were counted in the Laboratory within $8 \mathrm{~h}$ after sampling using a stereomicroscope and ranked according to the following density score: 0: no mites; 1: 1-5 mites; 2: 6-25 mites; 3: 26-50 mites; 4: 51-100 mites; 5: > 100 mites.

\subsection{Analytical procedures}

\subsubsection{IVM chemical extraction and chromatographic conditions}

IVM concentrations in plasma and skin samples were measured following an adaptation of the technique described by Lifschitz et al. (1999, 2000). Briefly, a $0.25 \mathrm{~mL}$ aliquot of plasma sample or $0.25 \mathrm{~g}$ of skin were combined with $20 \mathrm{ng}$ of the internal standard compound (moxidectin) and then mixed with $1 \mathrm{~mL}$ of acetonitrile. After mixing for $20 \mathrm{~min}$ (samples were sonicated in an ultrasonic bath for $10 \mathrm{~min}$. (Transsonic 570/H, Laboratory Line Instruments Inc., Melrose Park, IL, USA). The solvent-sample mixture was centrifuged at $2000 \times g$ during $15 \mathrm{~min}$ and the supernatant was manually transferred into a tube and concentrated to dryness under a stream of nitrogen. The derivatization of IVM was done following the technique described by De Montigny et al. (1990). IVM concentrations in plasma and skin were determined by HPLC using a Shimadzu 10A HPLC system with fluorescence detection reading at $365 \mathrm{~nm}$ (excitation) and $475 \mathrm{~nm}$ (emission wavelength). Calibration curves were prepared in the range between 0.2 and $100 \mathrm{ng} / \mathrm{mL}$ or g. Correlation coefficients (r) and coefficient of variations (CV) were calculated. The linear regression lines for IVM showed correlation coefficients $\geq 0.99$. The mean recoveries of IVM from plasma and skin were 76 and $91 \%$, respectively. The precision of the analytical method of IVM showed a CV of $6 \%$ and $4 \%$ for plasma and skin, respectively. The limit of quantification in plasma and skin was established at $0.2 \mathrm{ng} / \mathrm{mL}$ or $\mathrm{g}$.

\subsection{Pharmacokinetic and statistical analysis of the data}

The plasma and skin concentrations $v$ s. time curves obtained after each treatment in each individual animal were fitted with the PK Solutions 2.0 (Ashland, Ohio, USA) computer software. The area under the concentration $v s$. time curves (AUC) was calculated by the trapezoidal rule (Gibaldi and Perrier, 1982). IVM plasma concentrations and the pharmacokinetic parameters are reported as mean \pm SEM. Correlation between individual plasma and skin IVM concentrations was performed by parametric analysis (Pearson's $r$ ).

Mean IVM concentrations in plasma and skin and pharmacokinetic parameters were statistically compared using ANOVA. The assumption that the data obtained after treatments have the same variance was assessed. A Kruskal Wallis test was used where significant differences among standard deviations were observed. A value of $P<0.05$ was considered statistically significant. The statistical analysis was performed using the Instat 3.0 Software (Graph Pad Software, CA, USA). 
Parasite scores were calculated and expressed as mean \pm SEM. For each treatment, each animal served as its own control and the different scores were compared using Friedman test. Kruskal Wallis test was used to compare the scores obtained after different treatments. Based on cured animals in treated groups, the efficacy was calculated according to the following formula (Losson and Lonneux, 1996):

Efficacy (\%) from day 0 to day $x=$ (Total number of treated cattle - number of cattle still infected on day $\mathrm{x} /$ total number of treated cattle) $\times 100$.

\section{Results}

\subsection{Pharmacological assay}

IVM was detected in plasma and skin of all treated animals. Both commercial formulations showed similar levels of IVM plasma concentrations. Mean IVM concentrations for both formulations were between 22.5 and $2.8 \mathrm{ng} / \mathrm{mL}$ after single administration on day 0 (Groups 1 and 3) and between 44 and $6 \mathrm{ng} / \mathrm{mL}$ after the administration of IVM on days 0 and 7 (Groups 2 and 4). The comparative IVM plasma concentrations after the administration of both formulations are shown in Fig. 1. The repeated administration of both IVM formulations at day 0 and 7 (Groups 2 and 4) accounted for greater plasma drug concentrations resulting in higher IVM concentrations between 14 and 28 days post-treatment compared with the single administration $(\mathrm{P}<0.05)$. The mean AUC in plasma after the repeated administration of IVM was between 1.65 (Formulation A) and 2 (Formulation B) fold greater than those obtained after the single treatment.

IVM was well distributed from the plasma to the skin without significant differences between both formulations. There was a positive correlation between IVM concentrations in skin and plasma (r: $0.73 \mathrm{P}<0.0001$ ). The mean ratios between IVM availabililty (measured as AUC) in the skin and in plasma were between 1.2 and 2.1. The double administration of IVM increased significantly the IVM concentrations in the skin of areas affected by mange. The concentrations of IVM in the skin are compared in Fig. 2. Comparative mean IVM plasma and skin availability (measured as AUC) for formulations A and $\mathrm{B}$ are shown in Table 1.

\subsection{Parasitological observations}

IVM failed to achieve a parasitological cure in the different groups of animals affected by mange. The failure was observed with both formulations (A and B) administered as single or repeated treatment. At

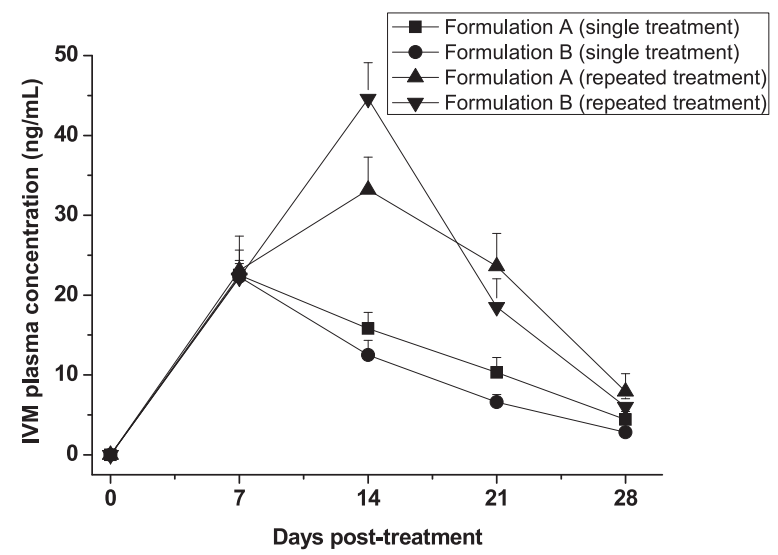

Fig. 1. Comparative mean $( \pm \operatorname{SEM})(n=8)$ ivermectin (IVM) plasma concentrations obtained after the subcutaneous administration (single and repeated treatments) of the formulations A and B to cattle naturally infected with Psoroptes ovis.

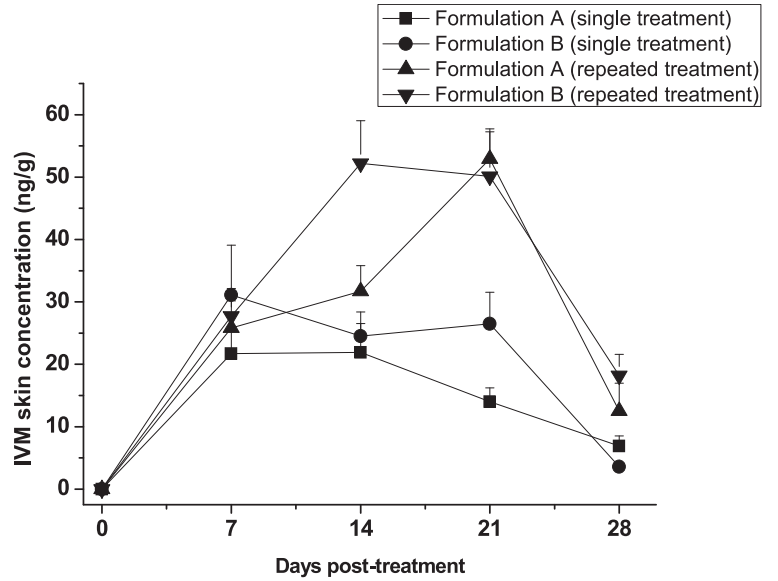

Fig. 2. Comparative mean ( \pm SEM) $(n=8)$ ivermectin $(I V M)$ skin concentrations obtained after the subcutaneous administration (single and repeated treatments) of the formulations A and B to cattle naturally infected with Psoroptes ovis.

Table 1

Comparative mean $( \pm$ SEM) $(n=8)$ ivermectin (IVM) plasma and skin exposure (measured as AUC) obtained after the subcutaneous administration (single and repeated treatments) of the formulations A and B to cattle naturally infected with Psoroptes ovis.

\begin{tabular}{llll}
\hline TREATMENT & $\begin{array}{l}\text { Plasma } \\
\text { AUC } \\
(\mathrm{ng} \cdot \mathrm{d} / \mathrm{mL})\end{array}$ & $\begin{array}{l}\text { Skin } \\
\text { AUC } \\
(\mathrm{ng} \cdot \mathrm{d} / \mathrm{g})\end{array}$ & $\begin{array}{l}\text { Ratio } \\
\text { AUC Skin/AUC } \\
\text { Plasma }\end{array}$ \\
\hline $\begin{array}{c}\text { Formulation A (single } \\
\text { treatment - day 0) }\end{array}$ & $355 \pm 25^{\mathrm{a}}$ & $429 \pm 50^{\mathrm{a}}$ & $1.22 \pm 0.14^{\mathrm{a}}$ \\
$\begin{array}{c}\text { Formulation B (single } \\
\text { treatment - day 0) }\end{array}$ & $300 \pm 30^{\mathrm{a}}$ & $587 \pm 75^{\mathrm{a}}$ & $2.10 \pm 0.32^{\mathrm{a}}$ \\
$\begin{array}{c}\text { Formulation A (repeated } \\
\text { treatment - days 0 and 7) }\end{array}$ & $586 \pm 83^{\mathrm{b}}$ & $812 \pm 122^{\mathrm{b}}$ & $1.42 \pm 0.11^{\mathrm{a}}$ \\
$\begin{array}{c}\text { Formulation B (repeated } \\
\text { treatment - days 0 and 7) }\end{array}$ & $618 \pm 65^{\mathrm{b}}$ & $980 \pm 158^{\mathrm{b}}$ & $1.70 \pm 0.29^{\mathrm{a}}$ \\
\hline
\end{tabular}

Different letters between rows show significant differences at $\mathrm{P}<0.05$.

AUC: area under the concentration $v s$. time curve between the moment of drug administration and day 28 following administration.

the beginning of the trial, $P$. ovis density scores were between 3.8 and 4.3. A significant decrease in $P$. ovis density scores was observed at 21 and 28 days post-treatment following the single IVM treatment, and at 14, 21 and 28 days post-treatment in the animals having received the repeated administration. Based on the number of animals cured, the range of efficacy was between $0 \%$ on day 7 and $60 \%$ on day 28 posttreatment. No significant differences in the $P$. ovis density scores were observed after the treatment with both formulations as single or repeated injections The scores and the efficacies of the different treatments against $P$. ovis are shown in Table 2.

The pharmacokinetic-pharmacodynamic relationship for IVM was evaluated using the plasma systemic exposure (measured as AUC) and the time (days) during which IVM plasma concentrations were above $15 \mathrm{ng} / \mathrm{mL}(\mathrm{T}>15)$. These pharmacological parameters were related with the individual efficacy against mites (density score). The mean AUC and $\mathrm{T}>15$ in the animals that were negative at day 28 (density score 0$)$ were significantly greater $(P<0.05)$ than those observed in the positive animals having mites counts $>1$ (Fig. 3).

\section{Discussion}

Psoroptic mange is a highly relevant parasitic disease that affects mainly beef cattle and leads to marked economic losses (Lonneux et al., 1998). The combination of stress factors related to Psoroptic mange may increase the maintenance energy requirements of calves, even when $P$. 
Table 2

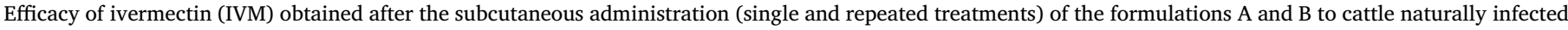
with Psoroptes ovis: mean density scores ( \pm SEM) and percentage of cured animals.

MEAN SCORE (\% Efficacy)

\begin{tabular}{|c|c|c|c|c|c|}
\hline TREATMENT & Day 0 & Day 7 & Day 14 & Day 21 & Day 28 \\
\hline $\begin{array}{l}\text { Formulation A } \\
\quad(\text { single treatment - day } 0)\end{array}$ & $4.1 \pm 0.3^{\mathrm{a}}(0)$ & $3.6 \pm 0.5^{\mathrm{a}}(0)$ & $2.6 \pm 0.5^{\mathrm{a}}(10)$ & $1.5 \pm 0.6^{\mathrm{b}}(40)$ & $1.4 \pm 0.4^{\mathrm{b}}(40)$ \\
\hline $\begin{array}{l}\text { Formulation B } \\
\quad(\text { single treatment }- \text { day } 0)\end{array}$ & $4.1 \pm 0.3^{\mathrm{a}}(0)$ & $3.3 \pm 0.5^{\mathrm{a}}(0)$ & $2.5 \pm 0.6^{\mathrm{a}}(10)$ & $2.0 \pm 0.7^{\mathrm{a}}(30)$ & $0.9 \pm 0.4^{\mathrm{b}}(50)$ \\
\hline $\begin{array}{l}\text { Formulation A } \\
\quad \text { (repeated treatment - days } 0 \text { and } 7 \text { ) }\end{array}$ & $4.3 \pm 0.3^{\mathrm{a}}(0)$ & $3.0 \pm 0.5^{\mathrm{a}}(10)$ & $2.6 \pm 0.6^{\mathrm{a}}(20)$ & $2.1 \pm 0.6^{\mathrm{b}}(30)$ & $1.1 \pm 0.3^{\mathrm{b}}(30)$ \\
\hline $\begin{array}{l}\text { Formulation B } \\
\quad \text { (repeated treatment - days } 0 \text { and 7) }\end{array}$ & $3.8 \pm 0.3^{\mathrm{a}}(0)$ & $2.8 \pm 0.4^{\mathrm{a}}(0)$ & $0.9 \pm 0.3^{\mathrm{b}}(50)$ & $0.8 \pm 0.2^{\mathrm{b}}(30)$ & $0.4 \pm 0.2^{\mathrm{b}}(60)$ \\
\hline
\end{tabular}

Density scores: 0: no mites; 1: 1-5 mites; 2: 6-25 mites; 3: 26-50 mites; 4: 51-100 mites; $5>100$ mites.

Different letters between columns (days post-treatments) show significant differences compared with the density score obtained at day 0 (P $<0.05$ ).

The values in brackets show the percentage of parasitologically cured animals at each day post-treatment.

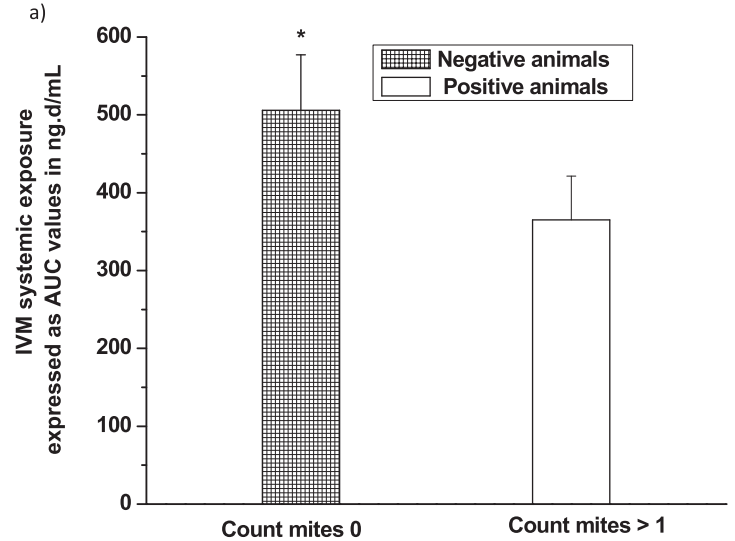

b)

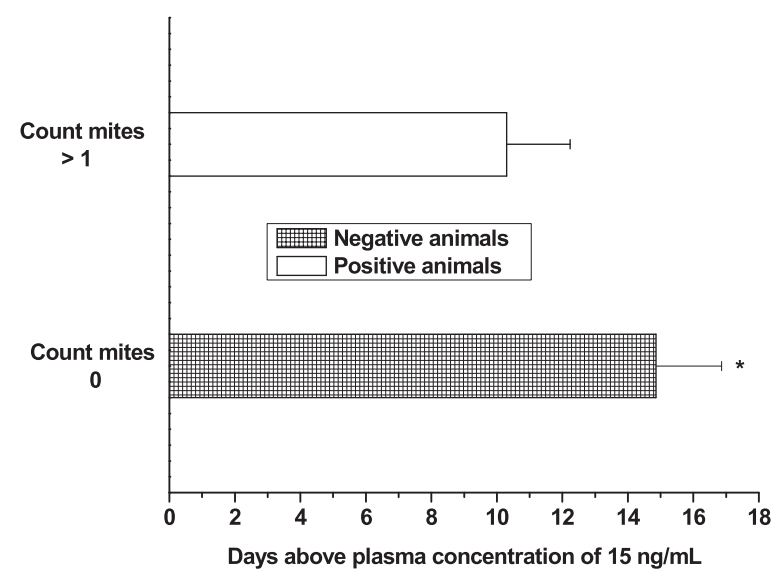

Fig. 3. Pharmacokinetic-pharmacodynamic relationship for ivermectin (IVM) against Psoroptes ovis: a) comparative mean plasma systemic exposure (measured as AUC) and b) time (days) during which IVM plasma concentrations were above $15 \mathrm{ng} / \mathrm{mL}(\mathrm{T}>15)$ obtained in the negative animals at day 28 post-treatment (density score 0 ) and in the positive animals with mites counts $\left.>1 .{ }^{*}\right)$ Mean values are significantly different $(\mathrm{P}<0.05)$.

ovis infestations are low (Cole and Guillot, 1987). Besides, infestations covering $30 \%$ of the body area have been shown to reduce the daily weight gain (Cole et al., 1984). Among the drugs available to control Psoroptic mange, IVM was widely chosen due to its excellent efficacy against $P$. ovis in cattle (Campbell and Benz, 1984). Many studies have reported that a single subcutaneous injection of IVM completely eliminates mite populations. However, the drug takes several days to become effective, and it does not kill all population of mites until 14 days after treatment (Guillot and Meleney, 1982). The physicochemical and pharmacokinetics features of IVM support its high efficacy against $P$. ovis. Due to its high lipophilicity, IVM shows a long persistence and a wide distribution from plasma to different tissues of parasite location which is essential for its activity against different parasites (Lifschitz et al., 2000).

In the current trial, IVM was detected in plasma from day 0 to day 28 post-administration. The repeated treatment at days 0 and 7 (Groups 2 and 4) accounted for a significantly higher systemic availability compared with that obtained after the single administration (Table 1). The distribution of IVM to the skin layers is relevant to achieve an optimal efficacy against $P$. ovis. Previous work has shown a strong correlation between plasma and skin concentrations of IVM after its subcutaneous injection to healthy cattle (Lifschitz et al., 2000). The ratio between IVM availability in the skin and plasma was determined as 1.45 (Lifschitz et al., 2000). The current work measures for the first time the relationship between plasma and skin concentrations of IVM in cattle affected by mange. This issue is very important as the inflammatory process and hyperkeratosis that exist in animals affected by $P$. ovis may lead to changes in the pattern of IVM distribution to the skin. In the current trial, IVM was widely distributed to the skin. Although the concentrations of IVM in skin correlated to those observed in plasma, the coefficient of correlation was lower (0.73) compared with that previously reported in healthy animals (0.99) (Lifschitz et al., 2000). The relationship between skin and plasma availability was between 1.22 and 2.10 , values that are in the same range than those observed in healthy calves (Lifschitz et al., 2000).

In this study, IVM failed to cure infected animals in contrast with a number of previous assays (Benz et al., 1989; Lonneux et al., 1997). In some cases, a repeated injection of IVM is needed in order to obtain an optimal efficacy, especially when animals suffer from hyperkeratosis and the drug is suspected to have more difficulties to reach the parasites (Vercruysse and Rew, 2002). Our study demonstrated high drug levels in the skin of affected animals, especially after the double injection of IVM. Therefore, the phamacokinetics should be discarded as the reason behind therapeutic failure. Although the density score of mites significantly decreased on day 28 post-treatment, the efficacy obtained after the administration of both IVM formulations as single or repeated treatments was between 30 and $60 \%$ (Table 2). Few reports on the failure of IVM treatment against $P$. ovis in cattle have been published (Genchi et al., 2008; Lekimme et al., 2010). In one of them, two treatments with a generic IVM formulation given at a 14-day interval failed to cure five out of eight Blue Belgian cattle and therefore a potential development of resistance was suspected (Lekimme et al., 2010). In another trial, although similar plasma kinetics disposition profiles were observed between the reference and generic formulations, only the animals treated with the pioneering pharmaceutical product were completely negative after two doses of IVM on days 0 and 8 (Genchi et al., 2008). In the current trial, the pioneer and the generic 
formulation showed equivalent IVM concentrations in plasma and skin of infected animals. Both formulations showed similar kinetics disposition profiles after the single and the repeated injection of IVM (Figs. 1 and 2, Table 1). However, both treatment protocols failed to control mange infections and animals in all of the experimental groups had living mites on day 28 post-treatment. The intensive use of IVM during the last 30 years, which in the Tandil area chiefly aims at controlling gastrointestinal parasites, could have exerted a selection pressure on mites towards resistance to IVM. Thus, failures in efficacy observed in the current trial may be due to the development of mite resistance against IVM. Recently, the treatment failure of sheep scab in United Kingdom has been associated to acquired resistance of $P$. ovis against ML. In this case, the lack of susceptibility of mites was corroborated in vitro (Doherty et al., 2018)

The characterization of the pharmacokinetic-pharmacodynamic relationship is relevant to optimize the use of antiparasitic compounds. This information is not usually available as the in vitro assays use extremely high concentrations compared with those observed in vivo. Lifschitz et al. (2000) suggested that $1 \mathrm{ng} / \mathrm{g}$ was the minimal drug concentration necessary to achieve an optimal efficacy against susceptible gastrointestinal nematodes. Based on this minimal IVM concentration, the period of time post-treatment during which IVM remains effective was estimated. In the case of ticks and other ectoparasites, the exposure to the drug following IVM injection occurs during feeding and therefore its persistence directly influences drug uptake and efficacy (Jackson, 1989). To achieve an efficacy of $99 \%$ against Rhipicephalus microplus, a minimal plasma concentration of $8 \mathrm{ng} / \mathrm{mL}$ during the whole tick development period (21 days) is considered appropriate (Davey et al., 2010). There is no similar information concerning $P$. ovis. Taking into account the efficacy and kinetics data, we hypothesized that $15 \mathrm{ng}$ / $\mathrm{mL}$ was the threshold plasma concentration necessary to cure animals infected with the particular $P$. ovis isolate involved in the current trial. The time above this minimal concentration was also calculated. The $\mathrm{T}>15$ was significantly longer in the animals without parasite counts (14.9 days) compared with the animals exhibiting living mites on day 28 (10.3 days) (Fig. 3). The AUC in the animals with a density score of 0 at day 28 post-treatment was significantly greater (40\%) compared with positive animals (Fig. 3). These preliminary data on the pharmacokinetic-pharmacodynamic relationship for the activity of IVM against $P$. ovis reinforce the relevance of the level and the length of drug exposure above the threshold for obtaining an optimal efficacy. Further in vivo and in vitro evaluations are needed to establish an accurate relationship between IVM concentrations and drug efficacy against $P$. ovis.

In Argentina, a low number of cattle mange outbreaks were reported for many years, mainly due to the intensive use of IVM to control gastrointestinal nematodes. However, veterinarians have reported an increase in mange infection cases in the last few years, as well as failure in the efficacy of IVM to control clinical outbreaks. Due to the high animal density and stress conditions, feedlots provide the ideal environment for mange development. The current trial reported for the first time failures of the efficacy of IVM against $P$. ovis. in Argentina, involving pharmacological records and parasitological observations. Although both commercial formulations showed a similar plasma kinetic disposition and high drug distribution to the skin, the level of drug exposure was not sufficient to cure the infected steers after both single and repeated administration of IVM. Additional studies are needed to confirm the presence of resistant strains of $P$. ovis and to establish appropriate measures to control the disease.

\section{Acknowledgements}

This research was supported by Consejo Nacional de Investigaciones Científicas y Técnicas CONICET and Agencia Nacional de Promoción Científica y Técnica (ANPCyT). We wish to express our gratitude to the staff of the feedlot Vanguardia Sur for their collaboration during the field activities, particularly to Vet. Mariano Gonzalez and Vet. Franco Ferrero. The revision of English style by the scientific translator Paula Viviani is greatly acknowledged.

\section{References}

Benz, G.W., Roncalli, R.A., Gross, S.J., 1989. Use of iverrnectin in cattle, sheep, goats, and swine. In: Campbell, W.C. (Ed.), Ivermectin and Abamectin. Springer-Verlag, New York, pp. 215-244.

Campbell, W., 2012. History of avermectin and ivermectin, with notes on the history of other macrocyclic lactone antiparasitic agents. Curr. Pharm. Biotechnol. 13 (853), 65 .

Campbell, W.C., Benz, G.W., 1984. Ivermectin: a review of efficacy and safety. J. Vet. Pharmacol. Ther. $7,1-16$

Cole, N.A., Guillot, F.S., 1987. Influence of Psoroptes ovis on the energy metabolism of heifer calves. Vet. Parasitol. 23, 285-295.

Cole, N.A., Guillot, F.S., Purdy, C.W., 1984. Influence of Psoroptes ovis (Hering) on the performance of beef steers. J. Econ. Entomol. 77, 390.

Cristel, S., Fiel, C., Anziani, O., Descarga, C., Cetrá, B., Romero, J., Fernández, S., Entrocasso, C., Lloberas, M., Medus, D., Steffan, P., 2017. Anthelmintic resistance in grazing beef cattle in central and northeastern areas of Argentina - an update. Vet. Parasitol. Reg. Stud. Rep. 9, 25-28.

Davey, R.B., Pound, J.M., Miller, J.A., Klavons, J.A., 2010. Therapeutic and persistent efficacy of a long-acting (LA) formulation of ivermectin against Rhipicephalus (Boophilus) microplus (Acari: Ixodidae) and sera concentration through time in treated cattle. Vet. Parasitol. 169, 149-156.

De Montigny, P., Shim, J.S.K., Pivnichny, J.V., 1990. Liquid chromatographic determination of ivermectin in animal plasma with trifluoroacetic anhydride and $\mathrm{N}$-methylimidazole as the derivatization reagent. J. Pharm. Biomed. Anal. 8, 507-511.

Doherty, E., Burgess, S., Mitchell, S., Wall, R., 2018. First evidence of resistance to macrocyclic lactones in Psoroptes ovissheep scab mites in the UK. Vet. Rec. 182, 106.

Eddi, C., Nari, A., Caracostantogolo, J., 2002. Use of macrocyclic lactones to control cattle parasites in south America. In: Vercruysse, J., Rew, R.S. (Eds.), Macrocyclic Lactones in Antiparasitic Therapy. Cabi Publishing, Wallingford, UK, pp. 262-287.

Genchi, C., Alvinerie, M., Forbes, A., Bonfanti, M., Genchi, M., Vandoni, S., Innocenti, M., Sgoifo Rossi, C.A., 2008. Comparative evaluation of two ivermectin injectable formulations against psoroptic mange in feedlot cattle. Vet. Parasitol. 158, 110-116.

Gibaldi, M., Perrier, D., 1982. Pharmacokinetics. Revised and Expanded, 2nd edn. Marcel Dekker, Inc., New York, USA.

Guillot, F.S., Meleney, W.P., 1982. The infectivity of surviving Psoroptes ovis (Hering) on cattle treated with ivermectin. Vet. Parasitol. 10, 73-78.

Jackson, H.C., 1989. Ivermectin as a systemic insecticide. Parasitol. Today 5, 146-156.

Kaplan, R.M., Vidyashankar, A.N., 2012. An inconvenient truth: global worming and anthelmintic resistance. Vet. Parasitol. 186, 70-78.

Lekimme, M., Farnir, F., Maréchal, F., Losson, B., 2010. Failure of injectable ivermectin to control psoroptic mange in cattle. Vet. Rec. 167, 575-576.

Lifschitz, A., Virkel, G., Pis, A., Imperiale, F., Sanchez, S., Alvarez, L., Kujanek, R. Lanusse, C., 1999. Ivermectin disposition kinetics after subcutaneous and intramuscular administration of an oil-based formulation to cattle. Vet. Parasitol. 86, 203-215.

Lifschitz, A., Virkel, G., Sallovitz, J., Sutra, J., Galtier, P., Alvinerie, M., Lanusse, C., 2000. Comparative distribution of ivermectin and doramectin to parasite location tissues in cattle. Vet. Parasitol. 87, 327-338.

Lonneux, J.F., Nguyen, T.Q., Losson, B.J., 1997. Efficacy of pour-on and injectable formulations of moxidectin and ivermectin in cattle naturally infected with Psoroptes ovis: parasitological, clinical and serological data. Vet. Parasitol. 69, 319-330.

Lonneux, J.F., Nguyen, T.Q., Detry, J., Farnir, F., Losson, B.J., 1998. The relationship between parasite counts, lesions, antibody titres and daily weight gains in Psoroptes ovis infested cattle. Vet. Parasitol. 76, 137-148.

Losson, B., Lonneux, J.F., 1996. Field efficacy of moxidectin 0.5\% pour-on against Chorioptes bovis, Damalinia bovis, Linognathus vituli and Psoroptes ovis in naturally infected cattle. Vet. Parasitol. 63, 119-130.

Vercruysse, J., Rew, R., 2002. General efficacy of the macrocyclic lactones to control parasites of cattle. In: Vercruysse, J., Rew, R.S. (Eds.), Macrocyclic Lactones in Antiparasitic Therapy. Cabi Publishing, Wallingford, UK, pp. 185-222.

Wright, F.C., 1990. Preliminary trials using a macrocyclic lactone against psoroptic scabies of cattle. Vet. Parasitol. 34, 289-294. 